\title{
Safety evaluation of Bifidobacterium pseudocatenulatum G4 as assessed in BALB/c mice
}

\begin{abstract}
Aims: To assess the safety of Bifidobacterium pseudocatenulatum G4 in BALB/c mice that involves examination of bacterial translocation, changes in the internal organs and histology of the intestinal lining. Methods and Results: Forty male BALB/c mice were randomly assigned into five groups $(\mathrm{n}=8)$. Three groups were orally fed with $50 \mu \mathrm{l}$ of three different concentrations of B. pseudocatenulatum G4 $(2 \times 104,1 \times 108$ and $1 \times 1011$ CFU day -1$)$ for 4 weeks. One group was orally administered with $50 \mu \mathrm{l}$ of $1 \times 108 \mathrm{CFU}$ B. longum BB536 per day for 4 weeks and last group was used as a nonbifidobacterial treatment control, which received $50 \mu \mathrm{l}$ of skim milk. The administered strains did not affect the general health of mice and incapable of carrying out translocation to blood or liver. There were no significant differences in the internal organ (liver, heart, kidney and spleen) indices, serum enzymes of liver (aspartate aminotransferase, alkaline phosphate, alanine aminotransferase) and kidney (urea and creatinine) and histology (villi height, crypts height, mucosa thickness and epithelial cell height) of caecum, ileum and colon. Conclusion: Administration of high dose of up to $1 \times 1011$ CFU B. pseudocatenulatum G4 per day to mice did not show any health threatening symptoms. Significance and Impact of the Study: Bifidobacterium pseudocatenulatum G4 is none pathogenic to BALB/c mice and could be safe probiotic for human consumption.
\end{abstract}

Keyword: Bifidobacterium, Infectivity, Marker enzymes, Organs, Safety, Translocation 